\title{
Comparison of General Anesthesia With Spinal Anesthesia on the Quality of Recovery of Patients With Selective Abdominal Hysterectomy in Patients Visiting the Largest Women's Disease Hospital in Northwestern Iran
}

\author{
Mir Mohammad Taghi Mortazavi ${ }^{1}{ }^{\mathbb{D}}$, Masoud Parish${ }^{1}$, Abbasali Dorosti ${ }^{1}$, Hassan Mohammadipour \\ Anvari $^{2 * \mathbb{D}}$
}

\begin{abstract}
Objectives: The quality of recovery can affect the results of abdominal hysterectomy although this effect is unknown in different methods of anesthesia. Therefore, the present study was conducted to compare general anesthesia (GA) and spinal anesthesia (SA) methods on the quality of the recovery of patients with selective abdominal hysterectomy in patients visiting the largest women's disease hospital in Northwestern Iran.

Materials and Methods: This cross-sectional descriptive study was conducted with the participation of 350 patients with abdominal hysterectomy, who were selected by convenience sampling in Al-Zahra hospital, Tabriz, Iran in 2019. Demographic data, visual pain scale, hemodynamic status, and Aldrete-Kroulik index were recorded for each patient. Data were analyzed using Mann-Whitney, t-test, multivariate regression, and Kolmogorov-Simonov tests in SPSS 20, and $P<0.05$ was considered statistically significant.

Results: In the hemodynamic status, it was found that the SA group was more stable than the GA group but this difference was not significant $(P>0.05)$. Regarding the need for pethidine, the results revealed that the mean ( \pm standard deviation) of the GA group was significantly higher $(35.14 \pm 10.14)$ than that of the SA group $(20.15 \pm 05.25, P=0.039)$ while there were no significant differences between the two groups in the use of the antiemetic drug $(P=0.203)$. Finally, the evaluations of the quality of recovery showed that the mean \pm ) SD) of the quality of recovery in SA patients was significantly higher compared to GA patients $(P=0.015)$.

Conclusions: In general, the quality of recovery was higher in the SA compared to the GA regarding abdominal hysterectomy.

Keywords: Recovery, Hysterectomy, Spinal anesthesia, General anesthesia
\end{abstract}

\section{Introduction}

The removal of the uterus and cervix without removing the adnexa is called hysterectomy $(1,2)$. Depending on the patient's condition, general or spinal anesthesia (GA or SA) may be chosen in this regard (3-5). The benefits of anesthesia in this surgery include patient satisfaction and the surgeon's comfort when surgery may take a long time, and the benefits of SA are hemodynamic stability and reduced bleeding (6-8).

Aspiration pneumonia, nausea and vomiting, and possibly the need for mechanical ventilation after surgery and death are common anesthesia complications, and neurological damage, headache, and hypotension are also common complications of SA $(9,10)$. Complications such as pain, nausea and vomiting, and hemodynamic changes under optimal anesthesia should be minimized to achieve comprehensive satisfaction of the patient, physician, and the health system $(11,12)$.

The advantage of SA over GA is the reduced need for postoperative narcotic administration, which reduces respiratory and gastrointestinal complications (i.e., ileus and nausea and vomiting). However, patient satisfaction is the main condition and indication for performing SA. The advantages of GA over SA include reduced patient stress and elevated physician's comfort $(3,13)$.

The patients' recovery phase begins after the surgery. The patient will be more stable in the ward if his/her recovery is more stable. Therefore, this stage and the events that occur at this stage are highly important for the subsequent planning of patients $(14,15)$. Since the number of patients undergoing abdominal hysterectomy surgery is on the rise, and stable postoperative conditions can affect the surgery outcome, the effect of anesthesia on postoperative complications has not been established in these patients. Accordingly, the current study focused on comparing the two methods of GA and SA regarding the quality of the recovery of patients with selective abdominal hysterectomy in those who referred to the largest women's disease hospital in Northwestern Iran. The specific objectives of the study are based on comparing the 


\section{Key Message}

What is the current knowledge?

- Respiratory and gastrointestinal complications in SA are less than the effects of GA.

- The postoperative recovery phase plays an important role in surgical results.

What Is the New Here?

- 1. Pethidine is more commonly used in patients undergoing SA for abdominal hysterectomy compared to GA

- 2. The hemodynamic status in SA is more stable than in GA.

- 3. The quality of recovery in SA is higher compared to GA in patients undergoing abdominal hysterectomy.

hemodynamic status, pain intensity, opioid requirement, recovery time, pain, antiemetic drug requirements, and the quality of recovery in the two methods of GA and SA.

\section{Materials and Methods}

Study Design

The present descriptive-analytical study was performed in Al-Zahra hospital, which is affiliated to Tabriz University of Medical Sciences, in accordance with the inclusion and exclusion criteria in 2019. The minimum sample size was estimated according to the results of a similar study (16) and assuming that the quality of recovery was equal in both groups with GA and SA. Considering a 95\% confidence level and $80 \%$ study power, 40 people were estimated for each group. In this study, all people with the inclusion and exclusion criteria (the total number of participants was 350) were included to increase the validity of the study. Participants entered the study using a convenience sampling method, and attempts were made to include an identical number of participants in each group (GA and SA).

The inclusion criteria included women aged 35-70 years, in grades I and II in the ASA (American Society of Anesthesiologists), and a candidate for abdominal hysterectomy. On the other hand, the exclusion criteria were coagulation disorders, infection over the past three months, history of cardiovascular disease, respiratory disease, rheumatoid arthritis, diabetes mellitus and history of opioid and corticosteroid use, severe neurological disorders, and a history of severe migraine headaches and a difficult airway.

\section{General Anesthesia Procedure}

Before transferring the patient to the operating room, 500 $\mathrm{mL}$ of normal saline serum was intravenously injected for all patients within half an hour. Pre-medication was performed with $2 \mathrm{mg}$ of midazolam and $100 \mu \mathrm{g}$ of fentanyl. After pre-medication, $2 \mathrm{mg} / \mathrm{kg}$ of the weight of propofol, $1 \mathrm{mg} / \mathrm{kg}$ of the weight of lidocaine, and $0.5 \mathrm{mg} / \mathrm{kg}$ of the weight of atracurium were injected and then intubated with tube number 7 or 7.5 after 5 minutes. Anesthesia was maintained with isoflurane (1 to $1.5 \mathrm{MAC}$ ), oxygen, and $50 \% \mathrm{~N}_{2} \mathrm{O}$ gas. Atracurium was repeated every 45 minutes if necessary. Eventually, the effects of the relaxant drug were reversed using atropine and neostigmine.

Spinal Anesthesia Procedure

In general, $500 \mathrm{~mL}$ of normal saline serum was intravenously injected for all patients within half an hour before transferring the patient to the operating room. The SA was then performed with a 25-gauge Quincke spinal needle in a sitting position at L2-L3 or L3-L4 space, and 10 $\mathrm{mg}$ of bupivacaine $0.5 \%$ was injected with 20 micrograms of fentanyl $(0.2 \mathrm{~mL})$ within 5-10 seconds. Immediately, the patient was lying supine, and simultaneously, the uterus and the bed were moved 10-15 degrees to the left using oxygen. A surgical incision was made in the sensory block at the level of the T4-6 dermatomes. In the case of a decrease in systolic blood pressure to less than $90 \mathrm{mmHg}$ or a drop of more than $30 \%$ of baseline, simultaneously with an increase in the venous fluid injection rate, 50 micrograms of phenylephrine (intervention group) up to a total dose of 200 micrograms and 5-10 mg of ephedrine up to a total dose of $20 \mathrm{mg}$ were intravenously prescribed in the phenylephrine and ephedrine (control group) groups, respectively. The cases of hypotension and bradycardia as a low heart rate were excluded from the study. The patient was excluded from the study and other treatments were performed if blood pressure was not controlled with the above measures and the heart rate was less than 60 or more than 100 beats per minute (17).

\section{Recovery Phase}

After the operation, all patients were transferred to the recovery unit. The recovery time for at least half an hour and hemodynamic monitoring were performed every three minutes. The monitoring of the hemodynamic status included recording the heart rate, systolic and diastolic blood pressure, arterial oxygen saturation, the need for analgesic, nausea and vomiting, and the average dose of an antiemetic drug for each group. If the patient's condition was such that the effects of anesthesia did not disappear within half an hour or the level of SA did not disappear within half an hour, according to the diagnosis of the recovery specialist, the patient would remain in the recovery unit until the disappearance of the effects of anesthesia/spinal. Moreover, the patients would stay in this unit until the stabilization of their conditions if they were unstable. Patients who needed cardiopulmonary resuscitation or transfer to the intensive care unit were excluded from the study.

\section{Data Collection Tools}

The applied tools in this study were a checklist based on study objectives. The first part included demographic data such as age, weight, height, and body mass index 
(BMI), along with a visual analogue scale to determine the severity of pain (measured by the researcher's help when entering and discharging from recovery), and questions related to the study variables. The intended variables were arterial oxygen saturation, systolic and diastolic blood pressure, heart rate (recorded at the time of entering and discharging from recovery), the mean amount of injected pethidine (in milligrams), the mean duration of recovery (in minutes), the average score of the AldreteKroulik index, and the mean amount of the antiemetic drug (ondansetron). It should be noted that hemodynamic status monitoring was performed by a monitoring device that could measure the heart rate, blood pressure (systolic and diastolic), and arterial oxygen saturation.

To prevent possible bias, multiple researchers were applied to collect data while the main researcher was absent from the research process, including entering data and carefully reviewing the entered data by two other persons separately.

\section{Statistical Analysis}

The data were recorded using the pen and paper method by the research assistant, and then entered into the SPSS20 by the statistical consultant, and finally, two people separately reviewed the entered data at separate times. Data were analyzed using Mann-Whitney, multivariate regression, t-test, and Kolmogorov-Simonov tests, and $P<0.05$ was considered statistically significant.

\section{Results}

During the study, 403 patients underwent abdominal hysterectomy surgery, of whom 350 people were assessed due to meeting the inclusion criteria. These individuals were placed in two equal groups (175 participants in each of the GA and SA groups) and were evaluated without any problems (no need for intensive care unit, no cardiopulmonary resuscitation, and no patient with unstable conditions). Patients in both groups were compared in terms of demographic characteristics, and the distribution of samples was normal based on demographic characteristics (Table 1).

The monitoring of the hemodynamic status in the two groups showed that only the heart rate of the GA group $(89.45 \pm 15.29)$ at the time of entering recovery was significantly higher $(P=0.008)$ than that of the SA group $(72.25 \pm 11.36)$. No significant difference was noted regarding other variables although the SA group was more stable than the GA group (Table 2).

The results of pain intensity demonstrated that the mean $( \pm S D)$ of pain intensity was significantly higher $(P=0.002)$ in GA patients $(06.52 \pm 01.45)$ compared to SA patients $(2.55 \pm 00.50)$. Regarding the need for pethidine, it was found that the mean $( \pm S D)$ of the GA group (35.14 $\pm 10.14)$ was significantly higher $(P=0.039)$ than that of the SA group $(20.15 \pm 05.25)$ although there were no significant differences between the two groups in terms of antiemetic drug use $(P=0.203)$. However, antiemetic drug

Table 1. Demographic Characteristics of the Participants

\begin{tabular}{lcc}
\hline \multirow{2}{*}{ Variable } & \multicolumn{2}{c}{ Groups $(\mathbf{N}=350)$} \\
\cline { 2 - 3 } & GA (n=175), Mean \pm SD & AS (n=175), Mean \pm SD \\
\hline Age & $42.39 \pm 5.89$ & $43.09 \pm 4.99$ \\
Weight $(\mathrm{kg})$ & $83.45 \pm 10.46$ & $85.03 \pm 11.03$ \\
Height $(\mathrm{cm})$ & $172.29 \pm 11.29$ & $175.45 \pm 11.22$ \\
BMl $\left(\mathrm{kg} / \mathrm{m}^{2}\right)$ & $28.41 \pm 3.48$ & 0.411 \\
\hline
\end{tabular}

Note. GA: General anesthesia; SA: Spinal anesthesia; BMI: Body mass index.

${ }^{a}$ Applied test: Kolmogorov-Smirnov.

Table 2. Comparison of Hemodynamic Status at Different Times Between GA and SA Groups

\begin{tabular}{|c|c|c|c|}
\hline \multirow{2}{*}{ Variable } & \multicolumn{2}{|c|}{ Groups $(\mathrm{N}=350)$} & \multirow{2}{*}{$P$ Value $^{\mathrm{a}}$} \\
\hline & GA $(n=175)$ & AS $(n=175)$ & \\
\hline $\mathrm{HR}$ at the time of entering recovery & $89.45 \pm 15.29$ & $72.25 \pm 11.36$ & 0.008 \\
\hline HR in getting out of recovery & $72.11 \pm 10.29$ & $69.45 \pm 10.12$ & 0.119 \\
\hline HR during recovery & $75.15 \pm 12.41$ & $73.01 \pm 12.90$ & 0.412 \\
\hline SBP at the time of entering recovery & $129.55 \pm 21.45$ & $120.61 \pm 20.37$ & 0.119 \\
\hline SBP in getting out of recovery & $139.55 \pm 20.93$ & $126.15 \pm 20.11$ & 0.129 \\
\hline SBP during recovery & $123.25 \pm 15.33$ & $125.16 \pm 20.44$ & 0.108 \\
\hline DBP at the time of entering recovery & $79.43 \pm 6.95$ & $73.12 \pm 5.26$ & 0.119 \\
\hline DBP in the get out of recovery & $85.24 \pm 7.25$ & $76.25 \pm 6.66$ & 0.203 \\
\hline DBP during recovery & $80.61 \pm 8.18$ & $75.42 \pm 8.39$ & 0.209 \\
\hline $\mathrm{SPO}_{2}$ at the time of entering recovery & $95.45 \pm 3.18$ & $98.12 \pm 1.10$ & 0.089 \\
\hline $\mathrm{SPO}_{2}$ in the get out of recovery & $97.12 \pm 1.45$ & $98.45 \pm 1.11$ & 0.115 \\
\hline $\mathrm{SPO}_{2}$ during recovery & $96.95 \pm 1.45$ & $98.40 \pm 1.37$ & 0.101 \\
\hline
\end{tabular}

Note. GA: General anesthesia; SA: Spinal anesthesia; HR: Heart rate; SBP: Systolic blood pressure; DBP: Diastolic blood pressure.

a Applied test: Mann-Whitney U. 
use in the GA group $(04.45 \pm 01.15)$ was higher compared to the SA group $(03.85 \pm 01.10)$. As regards the number of injections of pethidine, it should be noted that the group of patients with GA received this drug more than the group of patients with SA while the difference in the number of the injections of antiemetic drugs between the two groups was not significant (Figure 1).

Studies on the quality of recovery indicated that the mean $( \pm S D)$ of the quality of recovery in SA patients was significantly higher than that of GA patients $(P=0.015)$. Table 3 presents the mean $( \pm S D)$ of the quality of recovery at different recovery times. It was also found that the mean $( \pm$ SD) of recovery duration in SA patients $(32.51 \pm 5.25)$ was higher compared to patients with GA $(36.52 \pm 5.55)$ although it was not statistically significant. Based on the study of the quality of recovery based on the relevant index, patients in the SA group had a more stable condition compared to patients in the GA group at different times (Figure 2).

Considering that nausea and vomiting are of the most effective factors on the quality of recovery and their rates are high, the severity of recovery decreases regarding the relationship between the severity of nausea and the length of stay in recovery, hemodynamic status, age, medication requirement, the need for pethidine, and $\mathrm{BMI}$ as predictors of nausea and vomiting in the recovery section. In patients with SA, age and BMI are known to be predisposing factors for nausea and vomiting (Table 4).

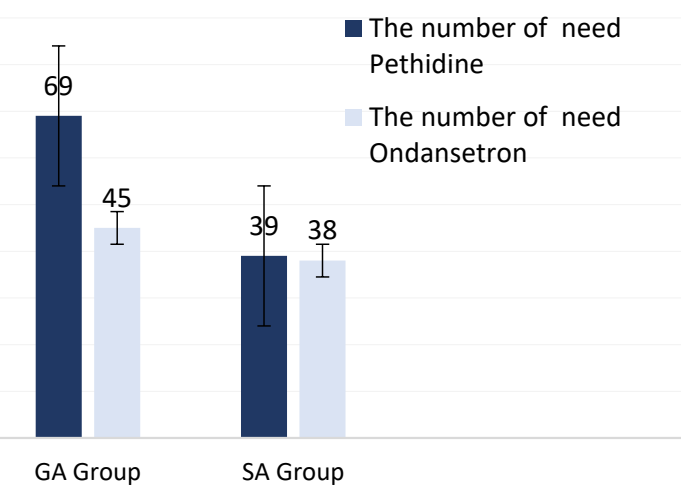

Figure 1. Comparison of Drug Use Frequency Between GA and SA Groups. Note. GA: General anesthesia; SA: Spinal anesthesia.

Table 3. Comparison of Recovery Quality at Different Times of Stay in Recovery Between the Two Groups Participating in the Study

\begin{tabular}{llll}
\hline Time in Recovery & GA $(\mathbf{n}=\mathbf{1 7 5})$ & $\mathbf{S A}(\mathbf{n = 1 7 5})$ & $\boldsymbol{P V a l u e}^{\mathrm{a}}$ \\
\hline The first five minutes & $03.12 \pm 0.15$ & $07.11 \pm 01.15$ & $0.001^{*}$ \\
The second five minutes & $04.15 \pm 0.45$ & $08.15 \pm 01.10$ & $0.003^{*}$ \\
The third five minutes & $05.85 \pm 01.10$ & $09.15 \pm 00.45$ & $0.001^{*}$ \\
Fourth five minutes & $06.40 \pm 00.55$ & $09.40 \pm 00.30$ & $0.008^{*}$ \\
Fifth five minutes & $08.15 \pm 02.30$ & $09.45 \pm 00.10$ & 0.069 \\
Fifth six minutes & $09.15 \pm 00.55$ & $09.55 \pm 00.40$ & 0.089 \\
\hline
\end{tabular}

Note. GA: General anesthesia; SA: Spinal anesthesia;

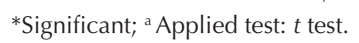

\section{Discussion}

The aim of this study was to compare the effects of GA and SA on the quality of recovery in patients with selective abdominal hysterectomy at the largest women's disease hospital in Northwestern Iran. The results of the present study showed that the recovery quality in SA was higher in comparison with GA. On the other hand, the length of patients' recovery stay in the SA group was less compared to the GA group. In a similar study (15), the study evaluated the quality of recovery in abdominal hysterectomy with GA and SA. Researchers reported that the quality of recovery after SA was higher than that of GA in women who were candidates for abdominal hysterectomy, which is in line with the results of the current study. Our study only focused on the quality of recovery during patients' stay in recovery while in the above-mentioned study, the quality of recovery was examined up to 2 days after the surgery. It seems that the effect of GA drugs and the negative effects of these drugs on various systems (e.g., respiratory, cardiovascular, nervous, and gastrointestinal systems) have led to a reduction in the quality of recovery in patients under GA. Many factors affect postoperative pain in the lower body in the recovery unit (18-20). On the other hand, following SA and nerve block in the lower extremity and the disappearance of this block after 3-6 hours, pain in recovery after SA is rarely observed, and therefore, these patients experience little pain. Finally, the quality of their recovery represents an increase.

Based on the examination of the hemodynamic status in the present study, among study variables, only the heart rate was higher in the first minutes after the surgery in the group undergoing GA and there was no difference between the two groups at other times. The increase in the heart rate in patients receiving GA could be due to neuromuscular blocking drug rivers and the effects of atropine, and it returned to a normal level by eliminating the effects of this drug. The results of several similar studies (21-23) revealed that there were significant statistical differences in the study variables such that the stability of hemodynamic status in SA was higher than

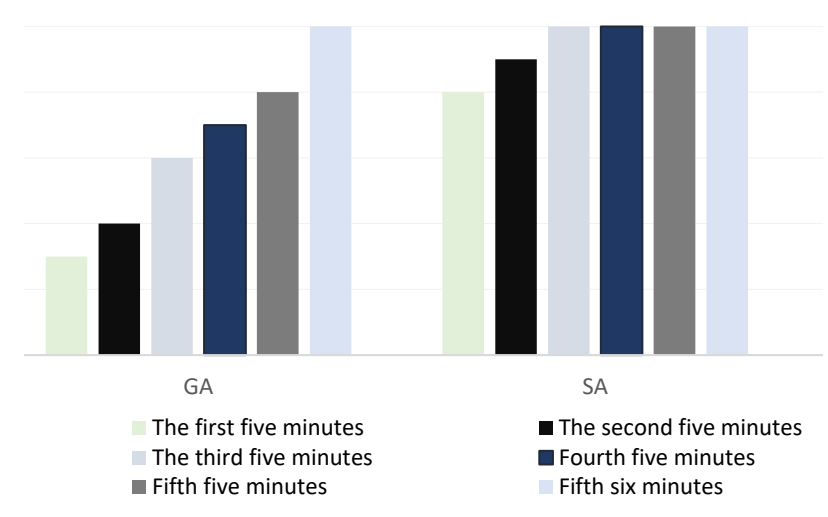

Figure 2. Comparison of Recovery Quality at Different Times Between GA and SA Groups. Note. GA: General anesthesia; SA: Spinal anesthesia. 
Table 4. Predictors of Nausea and Vomiting in the Two Groups Participating in the Study

\begin{tabular}{lcccc}
\hline \multirow{2}{*}{ Variable } & \multicolumn{2}{c}{ GA $(\mathbf{n}=\mathbf{1 7 5})$} & \multicolumn{2}{c}{ SA $(\mathbf{n}=\mathbf{1 7 5})$} \\
\cline { 2 - 5 } & $\boldsymbol{\beta}$ & $\boldsymbol{P}$ Value & $\boldsymbol{\beta}$ & $\boldsymbol{P}_{\text {value }}$ \\
\hline Age & 0.14 & 0.009 & 0.19 & 0.009 \\
BMI & 0.75 & 0.034 & 0.56 & 0.011 \\
Applied pethidine & 0.59 & 0.004 & 0.49 & 0.491 \\
Applied ondansetron & 0.215 & 0.122 & 0.39 & 0.109 \\
Duration of stay in the recovery unit & 0.25 & 0.213 & 0.49 & 0.211 \\
HR during recovery & 0.66 & 0.115 & 0.85 & 0.209 \\
SBP during recovery & 0.89 & 0.129 & 0.59 & 0.129 \\
SBP during recovery & 0.96 & 0.189 & 0.57 & 0.114 \\
SPO during recovery & 0.26 & 0.201 & 0.63 & 0.129 \\
\hline N
\end{tabular}

Note. GA: General anesthesia; SA: Spinal anesthesia; BMI: Body mass index;

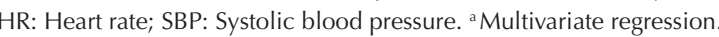

GA, which contradicts the findings of the current study. The hemodynamic status is highly related to the type of the surgery, the margins during the surgery, the amount of blood transfusion during the surgery, and the correct and sufficient fluid therapy during the surgery. All these factors should be considered during fluid therapy to reduce the complications of hemodynamic disorders with proper fluid therapy.

A study of the pain intensity in the present study demonstrated that patients in the GA group experienced more pain in recovery compared to patients in the SA group, and the need for analgesics in this group was naturally higher in comparison with the other group, which corroborates with the results of other studies $(24,25)$ It seems that other side effects of injectable medications during SA and the blockage of nerves in the lower extremities are the main reasons for the lack of an understanding of pain following this anesthesia. Therefore, the use of this method significantly reduces the severity of pain in the recovery unit, leading to a reduction in the need for analgesics. Pain is one of the main indicators of the quality of recovery after surgery and there is an inverse relationship between the severity of pain and the quality of recovery so that more severe pain results in lower quality of recovery. The results of this study represented that the quality of recovery in the SA group was higher than in GA.

The study of nausea and vomiting in patients participating in this study showed that there was no significant difference in the amount and frequency of antiemetic drug use between the two groups. However, the frequency of nausea/vomiting and the need for treatment were greater in the GA group compared to the SA group. In this regard, the results of similar studies $(26,27)$ indicated that GA causes more nausea and vomiting than other methods, increasing the need for antiemetic medications. Nausea and vomiting in surgeries (e.g., hysterectomy) can put pressure on the intestines and the lower gastrointestinal tract, which can have adverse effects on the results of hysterectomy. Therefore, the type of anesthesia method should be considered by anesthesiologists to minimize nausea and vomiting.

The results further indicated mass index, consumption pattern, and age as the predictors of nausea and vomiting in recovery for patients with GA and variables such as the $\mathrm{BMI}$ and age as the predictors of nausea and vomiting in recovery for patients in the SA group. Nausea and vomiting are the main variables affecting the quality of recovery and are directly related to the quality of recovery so that these two variables lead to a decrease in the quality of care during the recovery unit in patients. Recovery also has adverse effects on the results of surgery so that it can increase postoperative bleeding and complicate the surgical incision site. Therefore, the prevention of nausea and vomiting must be done at any stage after the surgery for safe care. The results of the present study demonstrated that age and BMI in both groups are the predictors of nausea and vomiting, thus preventive measures should be taken for all patients. The results of this study are consistent with those of other studies (28-32).

\section{Conclusion}

The quality of recovery in SA is higher compared to GA in abdominal hysterectomy.

\section{Limitations of the Study}

The lack of hemodynamic status monitoring and pain intensity assessment after discharge from the recovery unit, which could affect the quality of recovery in the first 24 hours, was the limitation of this study.

\section{Suggestions for Future Studies}

Researchers of the present study suggest further studies to evaluate the quality of recovery until patients are discharged from the hospital. The clinical findings of this study indicate that patients with SA are more stable than those undergoing GA, and anesthesiologists should use SA for candidates regarding abdominal hysterectomy.

Authors' Contribution

MMTM: Study design and intervention implementation; $M P$ and $A D$ : Intervention implementation and data collection; HMA: Manuscript preparation and article submission.

\section{Conflict of Interests}

Authors declare that they have no conflict of interests.

Ethical Issues

The ethical considerations observed in this study were obtaining ethical approval from the Ethics Committee of Tabriz University of Medical Sciences, explaining the objectives of the research to the participants in the study, and giving patients the right to choose the type of anesthesia method. The research project was approved by the Ethics Committee of Tabriz University of Medical Sciences (ethics no. IR.TBZMED.REC.1397.1059).

Financial Support

This study was granted by Tabriz University of Medical Sciences.

\section{Acknowledgments}

The researchers would like to give their gratitude to the Research Center and the Health Vice-chancellor of Tabriz University of Medical Sciences For financial support in the study. 


\section{References}

1. Lee SH, Oh SR, Cho YJ, et al. Comparison of vaginal hysterectomy and laparoscopic hysterectomy: a systematic review and meta-analysis. BMC Womens Health. 2019;19(1):83. doi:10.1186/s12905-019-07844

2. Khanbabaei Gol M, Aghamohamadi D. Effect of intravenous infusion of magnesium sulfate on opioid use and hemodynamic status after hysterectomy: double-blind clinical trial. Iran J Obstet Gynecol Infertil. 2019;22(7):32-38. doi:10.22038/ijogi.2019.13815

3. Munro A, Sjaus A, George RB. Anesthesia and analgesia for gynecological surgery. Curr Opin Anaesthesiol. 2018;31(3):274-279. doi:10.1097/aco.0000000000000584

4. Aghamohamadi D, Khanbabaei Gol M. An investigation into the effects of magnesium sulfate on the complications of succinylcholine administration in nulliparous women undergoing elective cesarean section: a double-blind clinical trial. Int J Womens Health Reprod Sci. 2019;7(4):520-525. doi:10.15296/ijwhr.2019.86

5. 5 Baghianimoghaddam $\mathrm{MH}$, Bakhtari-Aghdam F, Asghari-Jafarabadi $\mathrm{M}$, Allahverdipour H, Dabagh-Nikookheslat S, Nourizadeh R. The effect of a pedometer-based program improvement of physical activity in Tabriz University employees. Int J Prev Med. 2016;7:50. doi:10.4103/20087802.177897

6. Onafowokan O, Asemota O. Vaginal hysterectomy using local anesthesia and analgesics. Trop J Obstet Gynaecol. 2016;33(3):270273. doi:10.4103/0189-5117.199806

7. Kiatchai T, Sanansilp V, Triyasunant N, Saengprateep S, Changkittirat $\mathrm{P}$, Achariyapota $\mathrm{V}$. Effects of pregabalin on postoperative pain after hysterectomy under spinal anesthesia with intrathecal morphine: a randomized controlled trial. J Anesth. 2017;31(6):861-868. doi:10.1007/s00540-017-2406-3

8. Ashrey EM, Bosat BE. Impact of two different anesthetic techniques on immune response and postoperative pain for abdominal hysterectomy. Med J Cairo Univ. 2019;87(7):4363-4369. doi:10.21608/ mjcu.2019.78253

9. Liu J, Huang X, Hu S, Meng Z, He H. Individualized lung protective ventilation vs. conventional ventilation during general anesthesia in laparoscopic total hysterectomy. Exp Ther Med. 2020;19(4):30513059. doi:10.3892/etm.2020.8549

10. Biswas A, Gupta N, Kaushal S. Anesthetic management of a patient with Takayasu's arteritis undergoing total laparoscopic hysterectomy. Anaesth Pain Intensive Care. 2018;22(3):374-376.

11. Alas A, Hidalgo R, Espaillat L, Devakumar H, Davila GW, Hurtado E. Does spinal anesthesia lead to postoperative urinary retention in sameday urogynecology surgery? a retrospective review. Int Urogynecol J. 2019;30(8):1283-1289. doi:10.1007/s00192-019-03893-1

12. Naithani $U$, Verma D, Rajkumar S. Lower limb weakness and paresthesia after combined spinal epidural anesthesia for abdominal hysterectomy: a report of three cases. Anaesth Pain Intensive Care. 2015;19(2):187-191.

13. Steinberg $A C$, Schimpf $M O$, White $A B$, et al. Preemptive analgesia for postoperative hysterectomy pain control: systematic review and clinical practice guidelines. Am J Obstet Gynecol. 2017;217(3):303313.e6. doi:10.1016/j.ajog.2017.03.013

14. Rosenbaum AJ, Moore KJ, Louie M, Schiff LD, Carey ET. Postanesthesia care unit stay and complications after same-day discharge laparoscopic hysterectomy. J Gynecol Surg. 2020;36(2):47-53. doi:10.1089/ gyn.2019.0099

15. Deeds JL, Shaw PN, Elliot AR, Morgan BT. An anesthesia quality improvement plan to improve postoperative pain outcomes after hysterectomy. Anesthesia ejournal. 2016;4(1):34-42.

16. Alereza H, Banihashem N, Akhlaghi I, Amri P, Barat S. The effect of general anesthesia and spinal anesthesia on quality of recovery after abdominal hysterectomy; randomised clinical trial. J Adv Med Biomed Res. 2018;26(117):12-20. [Persian].
17. Fakhari S, Bile Jani I, Atashkhouei S, Khanbabayi Gol M, Soliemanzadeh S. Comparing the effect of hypotension treatment due to spinal anesthesia with ephedrine or phenylephrine on arterial blood gases and neonatal Apgar score during cesarean delivery in obese mothers: randomized clinical trial. Iran J Obstet Gynecol Infertil. 2019;22(10):12-20. doi:10.22038/ijogi.2019.14185

18. Alizadeh Sabeg P, Mehrabi E, Nourizadeh R, Poursharifi H, Mousavi $\mathrm{S}$. The effect of counseling on breast cancer awareness in rural Iranian women: a randomized controlled clinical trial. J Cancer Educ. 2019;34(6):1083-1091. doi:10.1007/s13187-018-1411-z

19. Nourizadeh R, Azami S, Farshbaf-Khalili A, Mehrabi E. The effect of motivational interviewing on women with overweight and obesity before conception. J Nutr Educ Behav. 2020;52(9):859-866. doi:10.1016/j.jneb.2020.04.219

20. Nazari B, Amani L, Ghaderi L, Khanbabayi gol M. Effects of probiotics on prevalence of ventilator-associated pneumonia in multitrauma patients hospitalized in neurosurgical intensive care unit: a randomized clinical trial. Trauma Mon. 2020;25(6):262-8.

21. Alereza H, Banihashem N, Akhlaghi I, Amrimale P, Barat S. Comparing the effect of general anesthesia and spinal anesthesia on postoperative pain after abdominal hysterectomy: a randomized clinical trial. J Mazandaran Univ Med Sci. 2018;28(159):130-136. [Persian].

22. Naithani U, Meena MS, Gupta S, Meena K, Swain L, Pradeep DS. Dose-dependent effect of intrathecal dexmedetomidine on isobaric ropivacaine in spinal anesthesia for abdominal hysterectomy: effect on block characteristics and hemodynamics. J Anaesthesiol Clin Pharmacol. 2015;31(1):72-79. doi:10.4103/0970-9185.150549

23. Harsten A, Kehlet H, Toksvig-Larsen S. Recovery after total intravenous general anaesthesia or spinal anaesthesia for total knee arthroplasty: a randomized trial. Br J Anaesth. 2013;111(3):391-399. doi:10.1093/ bja/aet104

24. Hoffman M, Hallett-Voss C, Devaiah G, Voss D. Fetal and Maternal Outcomes Following Cesarean Hysterectomy: A Comparison of General vs. Regional Anesthesia [6L]. Obstet Gynecol. 2016;127:99S. doi:10.1097/01.AOG.0000483415.76116.ed

25. Yazdi K, Ghorchaei A, Mozari S, Baghani F, Abdollahy AA, Behnampour N. Correlation of depth of anesthesia with hemodynamic parameters in patients undergoing elective abdominal surgery. J Res Dev Nurs Midwifery. 2015;12(2):16-20. [Persian].

26. Campos GO, de Jesus Martins M, Jesus GN, et al. Palonosetron versus ondansetron for prevention of nausea and vomiting after total abdominal hysterectomy under spinal anesthesia with intrathecal morphine: a double-blind, randomized controlled trial. BMC Anesthesiol. 2019;19(1):159. doi:10.1186/s12871-019-0830-7

27. Wang YM, Xia M, Shan N, et al. Pregabalin can decrease acute pain and postoperative nausea and vomiting in hysterectomy: A metaanalysis. Medicine (Baltimore). 2017;96(31):e7714. doi:10.1097/ md.0000000000007714

28. Saboktakin L, Rafeey M, Kousha A, Moradi SM. Study on prevalence of Helicobacterpylori infection in adolescents with failure to thrive to compare with control group. Life Sci J. 2012;9:1425-31.

29. Saboktakin L, Barzegar M, Hagh Jo A, Emamalizadeh M. Study on serum Copper and Zinc level of children with epilepsy during long term therapy with anticonvulsants. Life Sci J. 2012;9(4):1250-54.

30. Saboktakin L, Bilan N, Nikniaz A, Nazari M. Study on serum Leptin level of children with Asthma. Life Sci J. 2012;9(4).

31. Taghizadeh S, Haghdoost M, Mashrabi O, Zeynalikhasraghi Z. Antibiotic usage in intensive care units of Tabriz Imam Reza hospital, 2011. Am J Infect Dis. 2013;9(4):123-8.

32. Salehpour F, Bazzazi AM, Aghazadeh J, et al. Can serum glucose level in early admission predict outcome in patients with severe head trauma? World neurosurgery. 2016;87:132-5.

(C) 2022 The Author(s); This is an open-access article distributed under the terms of the Creative Commons Attribution License (http:// creativecommons.org/licenses/by/4.0), which permits unrestricted use, distribution, and reproduction in any medium, provided the original work is properly cited. 